27. Rovamo L, Nikkilä EA, Taskinen MR, Raivio KO 1984 Postheparin plasma lipoprotein and hepatic lipase activities in preterm newborns. Pediatr Res 18:1104

28. Schiff D, Chan G, Seccombe D, Hahn P 1979 Plasma carnitine levels during intravenous feeding of the neonate. J Pediatr 95:1043

29. Schmidt-Sommerfeld E, Penn D, Wolf H 1983 Carnitine deficiency in premature infants receiving total parenteral nutrition: effect of L-carnitine supplementation. J Pediatr 102:931

30. Taskinen MR, Tulikoura I, Nikkilä EA, Ehnholm C 1981 Effect of parenteral hyperalimentation on serum lipoproteins and on lipoprotein lipase activity of adipose tissue and skeletal muscle. Eur J Clin Invest 11:317

31. van Biervliet JP, Vinaimont N, Vercaemst R, Caster H, Rosseneu M 1981 Plasma apoprotein and lipid patterns in newborns: influence of nutritional factors. Acta Paediatr Scand 70:851

\title{
Newborn Sepsis following Antepartum Group B Streptococcal Maternal Infection in Rats
}

\author{
GARY J. NOEL, JOSE I. SANTOS, AND JOSEPH J. VITALE \\ Mallory Institute of Pathology, Boston City Hospital, Boston University School of Medicine, \\ Boston, Massachusetts 02118
}

\begin{abstract}
Group B streptococcus is an important pathogen in man and infection due to this bacteria is responsible for significant mortality and morbidity in neonates. An animal model of neonatal infection caused by group $B$ streptococcus that results from vertical transmission is described. Nine pregnant Sprague-Dawley rats received intraperitoneal inoculation of $10^{9}-10^{10}$ colony forming units of group B streptococcus on day 20 or 21 of gestation. Four of nine rats died following inoculation. A total of 51 pups was born to the surviving five mothers. Pups were sacrificed at 4- to 8-h intervals and cultures of blood, brain, liver, and spleen were obtained. Nineteen of 51 pups (37\%) had group B streptococcus isolated from blood or tissues within the first $48 \mathrm{~h}$ of life. Results suggest that antepartum systemic infection in rats can result in vertical transmission of disease. This animal model can be used to further study the mechanisms of transmission of group B streptococcus and the pathogenesis and treatment of neonatal sepsis caused by this pathogen. (Pediatr Res 19: 297-299, 1985)
\end{abstract}

\section{Abbreviations}

GBS, group B streptococcus

ip, intraperitoneal

CFU, colony forming units

Group B streptococcal infection occurs in two to three newborns per 1000 live births in the United States (1). Prophylactic intrapartum antibiotic regimens directed at interrupting transmission of GBS from mother to neonate has resulted in reduction of newborn colonization and infection (2-4). Difficulties in identifying women at risk for delivering infants who may develop group B streptococcal infections, however, have made indications for these regimens unclear.

Although an animal model for newborn group B streptococcal

Received August 24, 1984; accepted October 29, 1984.

Requests for reprints should be addressed to Jose I Santos, M.D., Mallory Institute of Pathology, 784 Massachusetts Avenue, Boston, MA 02118.

This research was supported in part by a grant from the Thrasher Foundation. sepsis in the rat has been established (5) and GBS surface colonization of pups born to mothers colonized with GBS has been demonstrated (6), a model in the rat that involves newborn sepsis resulting from maternal colonization of infection has not been described. The purpose of this study was to develop an animal model of vertical transmission of newborn sepsis following group B streptococcal challenge of mothers. A better understanding of the mechanisms of transmission of GBS and the pathogenesis of early-onset neonatal sepsis may help in our approach to new prophylactic and therapeutic regimens.

\section{MATERIALS AND METHODS}

Preparation of organisms. A human isolate of GBS type III further characterized as resistant to opsonization (7) was incubated in Todd-Hewitt broth (Difco Labs) at $37^{\circ} \mathrm{C}$ for $18 \mathrm{~h}$ and adjusted to $10^{8}-10^{9} \mathrm{CFU} / \mathrm{ml}$ in normal saline.

Animals. Pregnant outbred Sprague-Dawley rats (Charles River Labs) of 15-18 days gestation were obtained and housed in our animal quarters. On day 20 or 21 of gestation animals were given $2 \mathrm{ml}$ of GBS inoculum $\left(10^{9}-10^{10} \mathrm{CFU}\right)$ ip through a 25-gauge needle in the right upper quadrant of the abdomen. Care was taken not to inoculate viscera or uterus. Blood cultures obtained by incising the distal tail were taken at $2,24,48$, and $72 \mathrm{~h}$ following inoculation. Animals were closely observed and time of delivery was recorded. Soon after birth, and at regular intervals during the first $48 \mathrm{~h}$ of life, pups were sacrificed and cultures were obtained.

Isolation of $G B S$. Cultures were obtained from all dead adult females, stillborns, and sacrificed pups.

Blood for culture was obtained by aseptic intracardiac puncture with a 25 -gauge needle attached to a tuberculin syringe and inoculated onto blood agar plates which were incubated overnight at $37^{\circ} \mathrm{C}$.

Brain specimens were obtained by aseptic puncture of the cranium and aspiration with a 21-gauge needle attached to a tuberculin syringe. Brain tissue was inoculated onto blood agar plates and incubated.

Liver and spleen were aseptically removed from each animal upon opening the abdominal cavity. Care was taken not to incise the bowel or stomach and minimal bleeding was appreciated 
during this procedure. Tissue was homogenized in sterile Petri dishes and inoculated onto blood agar plates and incubated.

All colonies on blood agar plates consistent with GBS were subcultured and were considered to be GBS after demonstrating accentuation of hemolysis on blood agar when incubated with Staphylococcus aureus (CAMP phenomena).

\section{RESULTS}

Mothers. Nine pregnant dams were challenged with GBS (Table 1). Three received an inoculum of $1.8 \times 10^{9} \mathrm{CFU}$ (group 1) and 6 received $1 \times 10^{10} \mathrm{CFU}$ (group 2). All three animals in group 1 survived. Four animals in group 2 died within $78 \mathrm{~h}$ of inoculation without giving birth. These four animals were less active than surviving mothers and were noted to be tachypneic $24-48 \mathrm{~h}$ prior to their death. GBS was isolated from blood, brain, liver, and spleen of these four animals postmortem. Surviving mothers appeared active, fed well, and delivered litters within 26 to $54 \mathrm{~h}$ after inoculation with GBS. Surviving mothers appeared to care for their pups appropriately.

All mothers in group 1 were bacteremic at 2, 24, 48, and $72 \mathrm{~h}$ following challenge and thus were documented to be bacteremic with GBS prior to and after labor. In group 2, bacteremia was documented at $24 \mathrm{~h}$ following inoculation ( $26 \mathrm{~h}$ prior to delivery) in one surviving mother. The other surviving mother in group 2 had no organisms isolated from blood cultures taken 2,48 , and $72 \mathrm{~h}$ following inoculation. Blood culture was not obtained in this animal $24 \mathrm{~h}$ following inoculation because she was in labor.

Newborns. A total of 51 pups was born. Four were stillborn (three in group 1). Stillborn pups were not malformed or runted. Sixteen of 47 liveborn pups, and three of four stillborn pups had GBS isolated from blood or tissues. Four unborn pups from each

Table 1. Mortality and frequency of bacteremia following inoculation of pregnant rats with group B streptococcus

\begin{tabular}{lcc}
\hline & $\begin{array}{c}\text { Group 1 } \\
(n=3)\end{array}$ & $\begin{array}{c}\text { Group 2 } \\
(n=6)\end{array}$ \\
\hline Size of inoculum (CFU) & $1.8 \times 10^{9}$ & $1 \times 10^{10}$ \\
Mortality & $0 \%(0 / 3)$ & $67 \%(4 / 6)$ \\
Bacteremia & $100 \%(3 / 3)$ & $83 \%(5 / 6)^{*}$ \\
\hline
\end{tabular}

* Four animals had group B streptococcus isolated from postmortem blood culture.

Table 2. Frequency of isolation of GBS from blood and tissues in born pups

\begin{tabular}{lcc}
\hline & \multicolumn{2}{c}{ Group } \\
\cline { 2 - 3 } & 1 & 2 \\
& $(n=36)$ & $(n=15)$ \\
\hline Blood & $17 \%(6)$ & $13 \%(2)$ \\
Brain & $8 \%(3)$ & $20 \%(3)$ \\
Liver & $19 \%(7)$ & $33 \%(5)$ \\
Spleen & $19 \%(7)$ & $33 \%(5)$ \\
One or more source & $31 \%(11)$ & $53 \%(8)$ \\
\hline
\end{tabular}

Table 3. Frequency of isolation of GBS from born pups at time of sacrifice or death

\begin{tabular}{clll}
\hline & \multicolumn{2}{c}{ Group } & \\
\cline { 2 - 3 } Time (h) & \multicolumn{1}{c}{1} & \multicolumn{1}{c}{2} & \multicolumn{1}{c}{ Total } \\
\hline $0-4$ & $4 / 10(40 \%)$ & $2 / 4(50 \%)$ & $6 / 14(43 \%)$ \\
$4-12$ & $2 / 9(22 \%)$ & $1 / 2(50 \%)$ & $3 / 11(27 \%)$ \\
$12-24$ & $1 / 10(10 \%)$ & $3 / 6(50 \%)$ & $4 / 16(25 \%)$ \\
$24-36$ & $4 / 5(80 \%)$ & $1 / 1(100 \%)$ & $5 / 6(83 \%)$ \\
$36-48$ & $0 / 2(0 \%)$ & $1 / 2(50 \%)$ & $1 / 4(25 \%)$ \\
\hline
\end{tabular}

of the females that died prior to delivery had blood, brain, liver, and spleen cultures which were positive for GBS.

Table 2 summarizes the frequency of GBS isolation from blood and tissues in live-born and stillborn pups. GBS was most frequently isolated from liver and spleen homogenates. Two pups had GBS isolated from their spleen in the absence of other positive blood or tissue cultures. Four pups had GBS isolated from their liver in the absence of positive blood or tissue cultures.

Table 3 summarizes the frequency of GBS-positive cultures from pups with regard to hour of sacrifice or death. Of pups from whom GBS was isolated, six of $19(32 \%)$ had bacteria isolated from cultures obtained prior to $4 \mathrm{~h}$ of life. Five of these six pups had GBS isolated from their liver or spleen in the absence of positive blood or brain cultures. The other pup had GBS isolated from both liver and spleen in the absence of positive cultures of the blood or brain. Nine of 13 pups from whom GBS was isolated from cultures obtained after $4 \mathrm{~h}$ of life, had bacteria isolated from more than one source. All six positive blood cultures in newborn were obtained from pups sacrificed after 4 $\mathrm{h}$ of life.

Three pups died at 12-16 h of age before sacrifice (one in group 1). GBS was isolated from blood, brain, liver, and spleen of one of these animals (group 2). One pup died at $4 \mathrm{~h}$ of age (group 2); GBS was isolated from its spleen. GBS was not isolated from the other two pups.

\section{DISCUSSION}

Group B streptococcal infection in neonatal rats followed antepartum ip bacterial inoculation of mothers. It was apparent in our experiments that sublethal ip inoculation could establish maternal infection without affecting delivery or postpartum maternal-newborn interactions. Documentation of bacteremia in the majority of mothers within $2 \mathrm{~h}$ and persisting as long as 72 $\mathrm{h}$ following inoculation, suggests that maternal infection can be quickly and effectively produced after GBS ip challenge.

Thirty-seven percent (19/51) of pups born to GBS challenged mothers had GBS isolated from their blood or tissues. The frequency of newborn infection appeared to increase with the size of inoculum that mother received. It has been our experience that the isolation of bacteria from the blood, brain, liver, and spleen of newborn rats occurs following ip inoculation of their mothers and that isolation of GBS from blood and tissues is more sensitive than mortality in determining infection in the newborn rat. The greater frequency of isolation from liver and spleen when compared to blood (Table 2) suggests that isolation of GBS from liver and spleen does not solely reflect bacteremia and that bacteria may localize in these organs.

The frequency of isolation of GBS from pups at the time of sacrifice appears to be constant over the first $48 \mathrm{~h}$. Isolation of GBS from pups immediately after birth and from stillborns suggests that antepartum infection of newborns occurs in this model. Slightly higher frequencies of GBS isolation from pups greater than $24 \mathrm{~h}$ of age were noted (Table 3) and suggest that evolving infection may occur in the 1st day of life following vertical transmission.

The mechanism of vertical transmission of GBS in this model is not clear. Mothers did not appear to have abnormal or prolonged deliveries and it seems unlikely that ascending infection occurred in these animals. It may be that aspiration or swallowing of infected maternal blood during birth was a mode of transmission in some pups, however, this mechanism is not consistent with the isolation of GBS from pups immediately after birth or in stillborns. It is probable that antepartum infection occurred in pups in the absence of ascending infection which suggests that transplacental hematogenous transmission is important in this animal model. The pathogenesis of early-onset infection in human neonates born to colonized mothers with intact or recently ruptured membranes could be similar to that occurring in this animal model. Hematogenous transmission of GBS from mother 
to infant has been suspected as occurring, particularly in the presence of intact membranes (8). The importance of this mode of transmission of infection, however, is not well understood. It may be that antepartum maternal bacteremia increases the risk to the newborn for acquiring early-onset disease and that preventing newborn early-onset sepsis is dependent on effectively treating or preventing antepartum GBS maternal bacteremia.

Previously described animal models for GBS sepsis have included inoculation of bacteria into amniotic fluid (9) and directly into newborns $(5,10,11)$ and have not included maternal infection. This animal model of vertical transmission can be used to study the mechanisms of transmission of GBS from mother to infant and to investigate regimens aimed at preventing earlyonset group B streptococcal infection.

Acknowledgments. The authors thank Harry R. Hill, M.D. for reviewing this manuscript and Doris Gutierrez for her technical assistance.

\section{REFERENCES}

1. Fischer G 1983 Summary of the National Institute of Health workshop on group B streptococcal infection. J Infect Dis 148:163-166
2. Boyer KM, Gadzala CA, Kelly PD, Burd LI, Gotoff SP 1983 Selective intrapartum chemoprophylaxis of neonatal group B streptococcal early-onset disease. II. Predictive value of prenatal cultures. J Infect Dis 148:802-809

3. Boyer KM, Gadzala CA, Kelly PD, Gotoff SP 1983 Selective intrapartum chemoprophylaxis of neonatal group B streptococcal early-onset disease. III. Interruption of mother-to-infant transmission. J Infect Dis 148:810-816

4. Yow MD, Mason EO, Leeds LJ, Thompson PK, Clark DJ, Gardner SE 1979 Ampicillin prevents intrapartum transmission of group B streptococcus. JAMA 241:1245-1247

5. Ferrieri P, Burke B, Nelson J 1980 Production of bacteremia and meningitis in infant rats with group B streptococcal serotypes. Infect Immun 27:10231032

6. Ancona RJ, Ferriera P 1979 Experimental vaginal colonization and motherinfant transmission of group B streptococcus in rats. Infect Immun 26:599602

7. Santos JI, Shigeoka AO, Hill HR 1982 Strain differences in virulence of group B streptococci. Pediatr Res 16:347-350

8. Bergqvist G, Holmberg G, Rydner T, Vaclavinkova V 1978 Intrauterine death due to infection with group B streptococci. Acta Obstet Gynecol Scand $57: 127-128$

9. Larsen JW, London WT, Baker CJ, Curfman BL, Sever JL 1981 Intraamniotic infection due to group B streptococcus: treatment and antibody response. Obstet Gynecol 58:222-226

10. Furtado D 1976 Experimental group B streptococcal infections in mice: hematogenous virulence and mucosal colonization. Infect Immun 13:13151320

11. Vogel LC, Kretschmer RR, Padnos DM, Kelly PD, Gotoff SP 1980 Protective value of gamma globulin preparation against group $B$ streptococcal infections in chick embryos and mice. Pediatr Res 14:788-792

\title{
Humoral and Cell-Mediated Immunity in Growth Hormone-Deficient Children: Effect of Therapy with Human Growth Hormone
}

\author{
VAL ABBASSI AND JOSEPH A. BELLANTI \\ Division of Endocrinology, Department of Pediatrics and the International Center for Interdisciplinary Studies of \\ Immunology, Georgetown University School of Medicine, Washington, D.C. 20007
}

\begin{abstract}
To delineate the role of growth hormone (GH) in the development and function of the immune system, immunological parameters including quantitative immunoglobulins, T and B lymphocytes, phytohemagglutinin lymphoproliferative response and delayed hypersensitivity skin tests were studied in nine GH-deficient children prior to GH therapy and at 2 months and 9 to 12 months following therapy. The phytohemagglutinin response (74.1 \pm 37.6 , mean $\pm \mathrm{SD})$, and the $\mathrm{T}$ rosette $(58.3 \%$ $\pm 9.4)$, B rosette $(21.1 \% \pm 6.1)$, IgG $(810 \pm 241 \mathrm{mg} / \mathrm{dl})$, (IgA $140 \pm 85)$, and IgM (176 \pm 70$)$ levels in GH-deficient children were comparable to age adjusted values in normal children. Following GH therapy the phytohemagglutinin response increased significantly at 9 to 12 months posttherapy, 123.2 \pm 51.9 versus $74.1 \pm 37.6, p<0.05$. $T$ and $B$ rosettes, immunoglobulin concentrations, and hypersensitivity skin tests were not affected by GH therapy. Al-
\end{abstract}

Received June 15, 1984; accepted October 31, 1984.

Address for correspondence Val Abbassi, M.D., Department of Pediatrics Georgetown University Hospital, 3800 Reservoir Road, N.W., Washington, D.C. 20007. though an effect of GH was not demonstrable by these studies, a positive role of GH cannot be entirely excluded since total GH deficiency did not exist in all children. (Pediatr Res 19: 299-301, 1985)

\section{Abbreviations}

GH, growth hormone

PHA, phytohemagglutinin

GH plays a significant role in the normal development of thymic-dependent immune function in lower mammals $(3,4$, $11,14)$. Although immune deficiency states in man are frequently associated with impaired growth, abnormalities of $\mathrm{GH}$ secretion has not been implicated in such conditions. Moreover, the precise role of GH in the development and maturation of the thymic-dependent and thymic-independent immune functions in humans is as yet unknown. The purpose of the study 